[Bull. Agr. Chem. Soc. Japan, Vol. 22, No. 3, p. 157 162, 1958]

\title{
On the Susceptibility of the Crystalline and Retrograded Forms of Amylose of Different Molecular Weights toward Black-koji Amylase System
}

\author{
By Izue Yamasaki, Seinosuke UEda and Chikao Toda \\ Laboratory of Zymology and Industrial Fermentation, Department of Agricultural Chemistry, \\ Kyushu University, Fukuoka, Japan \\ Received January 6, 1958
}

\begin{abstract}
The crystalline and retrograded amylose preparations were digested by black-koji amylase system in the heterogeneous state such as in the case of raw starch digestion. It was found that the usual retrograded amylose should be fractionated into two parts-one easily digestible and the other resistant toward black-koji amylase system. The latter part, when recrystallized by $n$-butanol, however, turned highly susceptible toward the enzyme action. Its molecular weight, as determined by the light scattering method, was found to be 32,000 , a value much smaller than that of the original amylose $(140,000)$.
\end{abstract}

\section{INTRODUCTION}

There are many reports dealing with the retrograded starch and its resistance to enzyme action. As early as in 1903, Maquenne1) reported retrograded starch is not attacked by malt diastase hence, the extent of the retrogradation was determined by measuring the decrease in the amount of starch saccharified by malt amylase. Using a similar analytical technique, except substituting ptyalin for malt amylase, Sallinger ${ }^{2)}$ found that a solution of Lintner soluble starch retrograded rapidly at $7^{\circ} \mathrm{C}$. Whistler and Johnson ${ }^{3)}$ demonstrated that as the average molecular size of potato amylose was reduced by mild acid hydrolysis, the extent of retrogradation during a 24 hour-period increased to its maximum and later decreased. From this fact, they suggested that for amlyose molecules there exists a critical molecular size for which retrogradation rates are at a maximum. Molecules of a larger size than the critical retrograde more slowly, presumably being

1) L. Maquenne, Compt. rend., 137, 88, 797, 1266 (1903).

2) H. Sallinger, Kolloid Z., 25, 111 (1919).

3) R. L. Whistler and C. Johnson, Cereal Chem., 25, 418 (1948). due to the steric effects resulting from their more coiled or kinked structure. On the contrary, molecules of smaller size retrograde more slowly, presumably being due to the increased solubility inherent in a lower molecular weight molecule. Recently, Lansky et al.4) claimed that the tapioca A-fraction does not retrograde because its chain molecule is too long to permit ready orientation to an aggregated state. The molecular weight of those amyloses, however, was only surveyed by intrinsic viscosity and reducing value qualitatively.

On the other hand, one of the authorss) has reported on the digestion of various kinds of raw starch by black-koji amylase system, and found that the saccharogenic amylase fraction hydrolyzed raw glutinous starches easily, but only weakly in case of non-glutinous starches. This fact seems to suggest that there will exist some relationships between the existent conditions of amylose in the starch granule and its digestibility.

In the present paper, amyloses prepared in

\footnotetext{
4) S. Lansky, M. Kooi and T. J. Schoch, J. Am. Chem. Soc., 71, 4066 (1949).

5) S. Ueda, J. Agr. Chem. Soc. Japan, contribution.
} 
the crystalline and retrograded forms were subjected to digestion in the heterogeneous state, by black-koji amylase. Furthermore, the digestibility of amylose in both forms of different molecular weight were compared. The molecular weights of amyloses were mined by the light scattering method.

\section{EXPERIMENTAL}

Preparation of Black-koji Amylase Solution: As reported previously ${ }^{5)}$, black-koji amylase solution was prepared from the shaking culture of Aspergillus awamori (Kawachi strain).

Preparations of Amylose.

(1) Crystalline amylose: Crystalline amylose was prepared from potato starch by the "precipitantenzyme method" devised by Nikuni et al. ") After the addition of $2.2 \mathrm{ml}$ of $n$-caprylic acid to about $2 l$ of 2 per cent hot potato starch paste, it was cooled down to room temperature. One-tenth $\mathrm{N}$ acetate buffer, $\mathrm{pH} 4.8,(100 \mathrm{ml})$ and 0.6 per cent Taka-diastase $100 \mathrm{ml}$ were added to the starch paste and the paste digested for a period of 3 hours at room temperature. The enzymatic action was stopped by rapid heating and the digested solution, after it was added with active carbon, was filtered with suction in a hot state. On cooling the clear filtrate after further addition of $0.8 \mathrm{ml} n$-caprylic acid, disk-shaped crystals of amylosecaprylic acid complex separated out. After the crystals were collected by centrifugation and washed twice with $n$-butanol saturated water, they were dissloved in $2 l$ of distilled water and $200 \mathrm{ml}$ of $n$-butanol was added to the solution. After heating to boil, the solution was gradually cooled down to room temperature. The recrystallized complex (dia $6 \mu$ petal-shaped) was collected, washed, dried and weighed (Amylose-I). The yield of amylose was 7-8 per cent of the potato starch.

(2) Retrograded amylose: Five $g$ of Amylose-I was dissolved in $100 \mathrm{ml} 1 \mathrm{~N} \mathrm{NaOH}$ in the icebox. After filtration through glass filter No. 4 , the filtrate was neutralized with $1 \mathrm{~N} \mathrm{HCl}$ to about $\mathrm{pH} 5.0$ and allowed to retrograde for three days in the icebox. Then, the amylose retrograded and precipitated to the bottom. Collecting by centrifugation, the retrograded amylose was washed with cold distilled water until the iodine coloration of the washings disappeared (Retrograded Amylose-I).

6) Z. Nikuni, K. Takaoka and H. Fuwa, J. Agr. Chem. Soc. Japan, 26, 216 (1952).
This retrograded amylose was digested in the heterogeneous state by black-koji amylase solution until the digestion proceeded no more, and the remainder was washed and dried (Retrograded Amylose-II).

This Retrograded Amylose-II was treated once more by dissolving in alkali and retrograding in the icebox (Retrograded Amylose-III).

(3) Crystallization of retrograded amylose: Retrograded amylose could not be crystallized by the method usually employed because of its instantaneous precipitability in the non-alkaline solution. The following device gave way to successful crystallization of retrograded amylose. After dissolving $1 \mathrm{~g}$ of dry powder of the Retrograded Amylose-II in $250 \mathrm{ml} \mathrm{IN}$ $\mathrm{NaOH}$, the solution was filtered through glass filter No. 4 and neutralized with $1 \mathrm{~N} \mathrm{HCl}$ which was previously added with one-tenth in volume of $n$-butanol in order to avoid the momentary precipitation of amylose. After adding one-tenth in volume of $n$-butanol, the whole solution was first heated to boil and gradually cooled down to room temperature for a period of six hours. Thus, rectangular crystals of amylose-butanol complex $(11 \times 7 \mu)$ were obtained.

Digestion of Retrograded and Crystalline Amyloses by Black-koji Amylase System.

(1) Action on Retrograded Amylose.

(a) Heterogeneity of Retrograded Amylose:

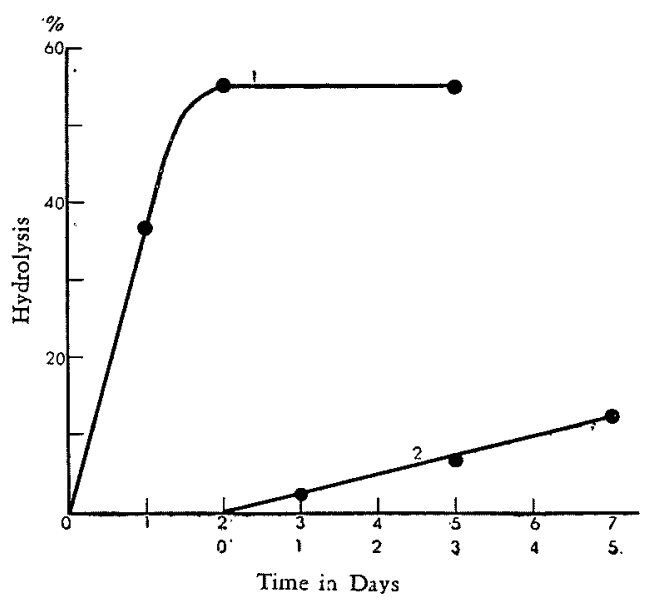

FIG. 1. Hydrolysis of Retrograded Amylose by Black-koji Amylase System.

Curve 1; Hydrolysis-time curve of the retrograded amylose prepared directly from Amylose-I.

Curve 2; Hydrolysis-time curve of the re-retrograded amylose prepared from resistant half to black-koji amylase of retrograded amylose. 
Amylose-I (0.1g) was dissolved in $5 \mathrm{ml} 1 \mathrm{~N} \mathrm{NaOH}$ and neutralized with $1 \mathrm{~N} \mathrm{HCl}$ and placed in the icebox for three days. The amylose retrograded and precipitated on the bottom of the flask. To this flask which contained retrograded amylose in the precipitated form, were added black-koji amylase solution $5 \mathrm{ml}$, M/5 citrate buffer ( $\mathrm{pH} 3.6$ ) $5 \mathrm{ml}$ and toluene $1 \mathrm{ml}$ and the reaction mixture incubated at $30^{\circ} \mathrm{C}$ for digestion. As shown in Fig. 1, the hydrolysis-time curve showed a distinct break at about 50 per cent hydrolysis (curve 1). In order to investigate whether the remained retrograded amylose was inherently resistant or became resistant during the digestion with black-koji amylase, the following experiment was carried out. After Amylose-I was retrograded and digested by black-koji amylase for two days as described above, the remained retrograded amylose was collected, washed, dissolved in $5 \mathrm{ml} 1 \mathrm{~N} \mathrm{NaOH}$, neutralized with $5 \mathrm{ml} 1 \mathrm{~N} \mathrm{HCl}$ and retrograded once more in the icebox for three days. To this retrograded amylose suspension, were added $5 \mathrm{ml}$ of black-koji amylase solution, $5 \mathrm{ml}$ of $\mathrm{M} / 5$ citrate buffer ( $\mathrm{pH} 3.6$ ) and $1 \mathrm{ml}$ of toluene and the reaction mixture similarly incubated at $30^{\circ} \mathrm{C}$ as before.

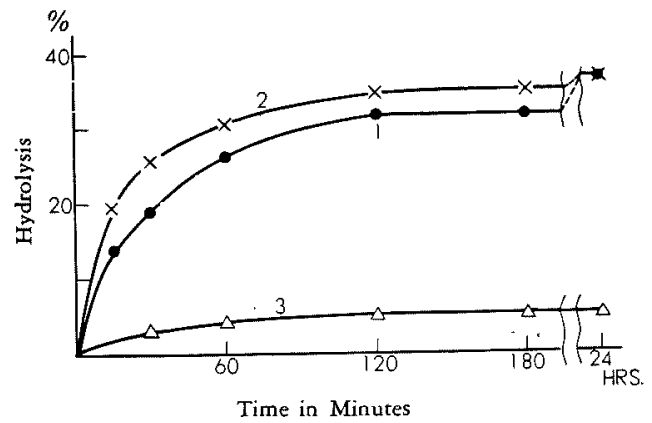

FIG. 2. Hydrolysis of Retrograded Amylose by Black-koji Amylase System.

Curve 1; Retrograded Amylose-I.

Curve 2; Retrograded Amylose-I. (The concentration of the amylase was used twice in the Curve 1.)

Curve 3; Retrograded Amylose-III.

As shown in curve 2 in Fig. 1, the re-retrograded amylose was found to be extremely resistant against black-koji amylase. From these results it is plausible to consider that the retrograded amylose should be fractionated into two fractions, one which is easily digested by black-koji amylase, the other very resistant to the amylase.

(b) Action of Black-koji Amylase on Retrograded Amylose-I and -III: Retrograded Amylose-I was directly prepared from Amylose-I. Retrograded Amylose-III was prepared by re-retrogradation of the resistant half after digesting Retrograded Amylose-I with black-koji amylase.

The compositions of the reaction mixtures were as follows :

Reaction mixture I

Retrograded Amylose-I

$10 \mathrm{ml}$ (amylose $134.0 \mathrm{mg}$ as glucose)

Black-koji amylase solution $5 \mathrm{ml}$

$\mathrm{N} / 10$ acetate buffer ( $\mathrm{pH} 3.6$ ) $\quad 10 \mathrm{ml}$

Distilled water

5. $\mathrm{ml}$

Reaction mixture II

Retrograded Amylose-I

$10 \mathrm{mI}$

(amylose $134.0 \mathrm{mg}$ as glucose)

Black-koji amylase solution

$10 \mathrm{ml}$

$\mathrm{N} / 10$ acetate buffer $(\mathrm{pH} 3.6$ )

$10 \mathrm{ml}$

Reaction mixture III

Retrograded amylose-III $5 \mathrm{ml}$

(amylose $31.0 \mathrm{mg}$ as glucose)

Black-koji amylase solution $\quad 5 \mathrm{ml}$

$\mathrm{N} / 10$ acetate buffer $(\mathrm{pH} 3.6) \quad 5 \mathrm{ml}$

These reaction mixtures were incubated at $30^{\circ} \mathrm{C}$ for digestion. As shown in Fig. 2, the rate and extent of hydrolysis of Retrograded Amylose-I showed a distinct break at 30 per cent hydrolysis and afterwards the digestion proceeded very slowly up to 35 per cent (curves 1 and 2). Much difficulty was found in hydrolysis of Retrograded Amylose-III and the extent of hydrolysis amounted up to only 5 per cent. These facts again confirmed that the retrograded amylose could be separated into two fractions, i. e., the resistant and the non-resistant to black-koji amylase. Difference in the extent of hydrolysis in Figs. 1 and $2(50 \%$ and $35 \%)$ seems to be attributed to the loss of digestible retrograded amylose by the washing of Retrograded Amylose-I in the case of Fig. 2.

(2) Action of Black-koji amylase on the Crystalline Amylose.

Grystalline Preparations from Amylose-I and those from Retrograded Amylose-II represented different shapes of crystals as shown in photographs 1 and 2 .

The compositions of the reaction mixtures were as follows :

Reaction mixture I

Crystalline Amylose-I suspension $\quad 5 \mathrm{ml}$

$\mathrm{N} / 10$ acetate buffer ( $\mathrm{pH} \mathrm{3.6)} \quad 5 \mathrm{ml}$

Distilled water ( $n$-butanol saturated) $4 \mathrm{ml}$

Black-koji amylase solution $\quad 5 \mathrm{ml}$

$n$-butanol $1 \mathrm{ml}$ 


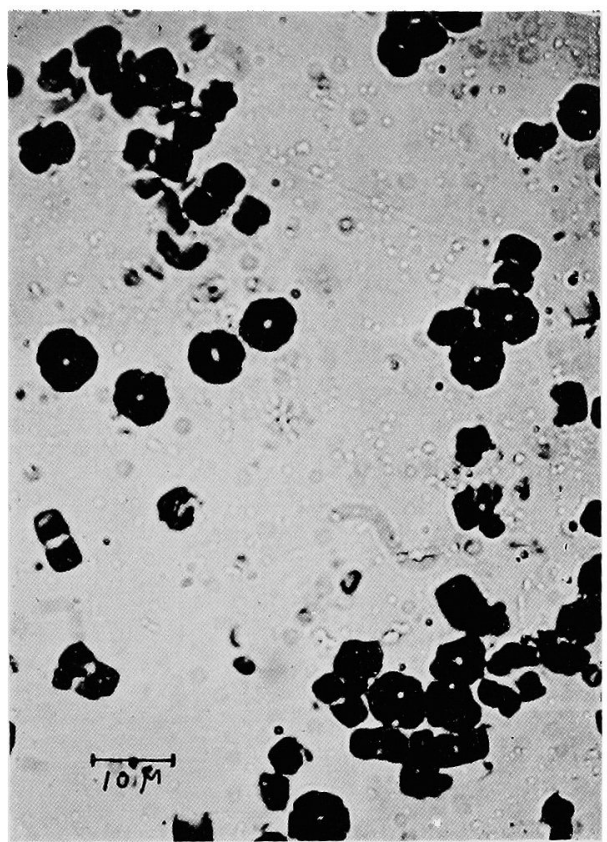

Photograph 1. Crystalline Amylose-Butanol Complex from Amylose-I.

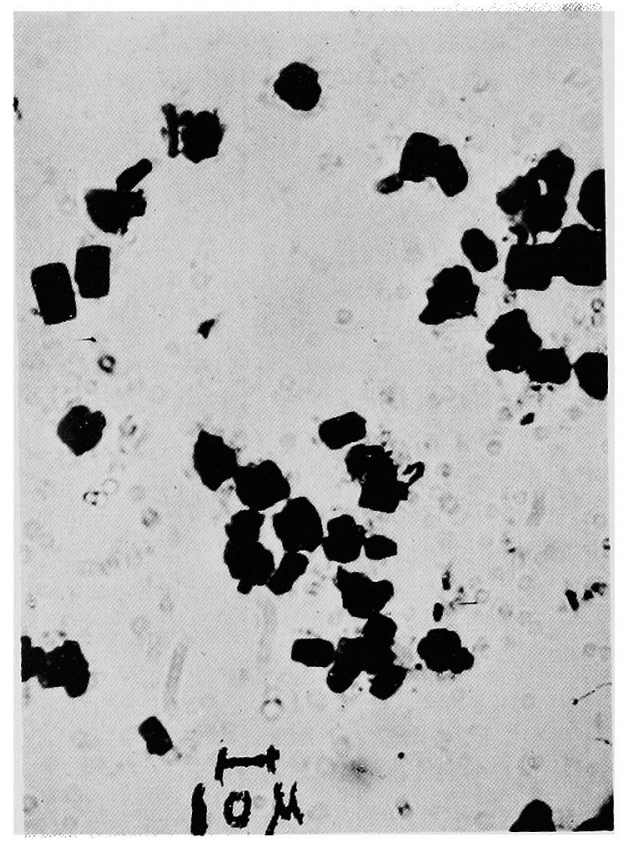

Photograph 2. Crystalline Amylose-Butanol Complex from Retrograded Amylose-II.
Reaction mixture II

Amylose solution*

$10 \mathrm{ml}$

$\mathrm{N} / 10$ acetate buffer $(\mathrm{pH} 3.6)$

$5 \mathrm{ml}$

Black-koji amylase solution

$5 \mathrm{ml}$

Reaction mixture III

Crystalline amylose

from Retrograded Amylose-II $\quad 10 \mathrm{ml}$

$\mathrm{N} / 10$ acetate buffer $(\mathrm{pH} 3.6) \quad 10 \mathrm{ml}$

Black-koji amylase solution $\quad 8 \mathrm{ml}$

$n$-butanol

$2 \mathrm{ml}$

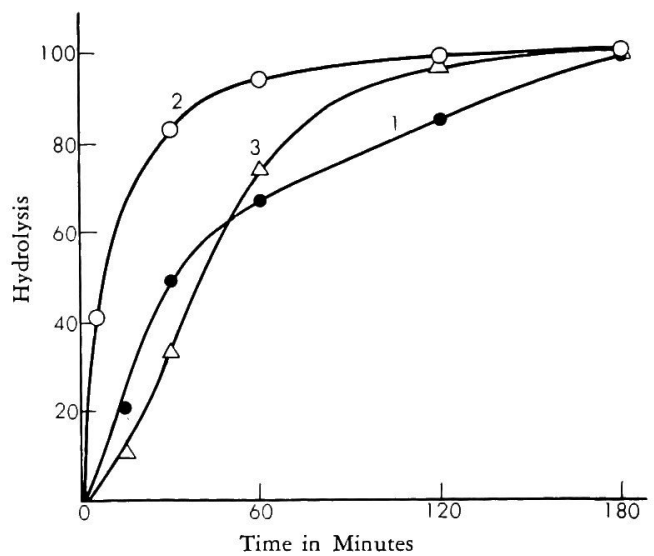

FIG. 3. Hydrolysis of Crystalline Amylose by Black-koji Amylase System.

Curve 1; Hydrolysis of the suspension of crystalline amylose prepared from Amylose-I.

Curve 2; Hydrolysis of the solution of crystalline amylose prepared from Amylose-I.

Curve 3; Hydrolysis of the suspension of crystalline amylose prepared from Retrograded Amylose-III.

As shown in Fig. 3, the crystalline amylose (curves 1 and 3) was converted to glucose as smoothly as amylose solution (curve 2), though the rates of hydrolysis slightly differed from each other.

It is very interesting to note that retrograded amylose, which could not be attacked by the blackkoji amylase in the retrograded state, changed to be highly susceptible to the enzymic action in the crystalline state.

Determination of Molecular Weights by the Light Scattering Method.

Molecular weights of Amylose-I and of Retrograded Amylose-II were determined by this method.

Amylose preparation was dissolved in $\mathrm{N} / 3 \mathrm{NaOH}$. After centrifugation for 2 hours at 12,000 r.p.m.

* Five $\mathrm{ml}$ of distilled water was added into $5 \mathrm{ml}$ crystalline amylose suspension to dissolve the crystals, 


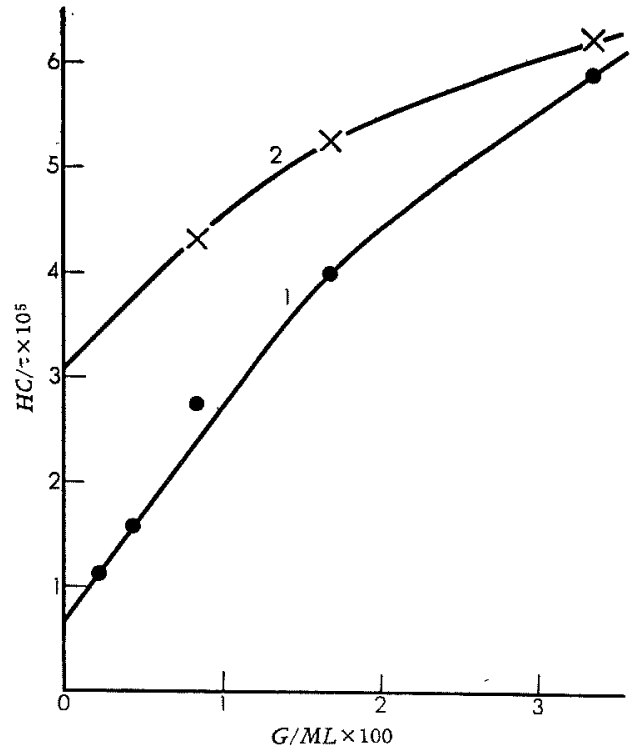

FIG. 4. Determination of $\underset{C \rightarrow 0}{H C / \tau}$ by the Light Scattering Method.

Curve $1 ; H C / \tau-C$ curve of Amylose-I.

Curve 2; $H C / \tau-C$ curve of Retrograded Amylose-II.

$(14,500 \times \mathrm{g})$ in order to remove the dust, the supernatant was used for measuring the refractive index* and the intensity of light scattering**.

Molecular weight was calculated for each preparation, using the following equation derived by Debye ${ }^{7)}$

$$
\begin{aligned}
& M=\frac{\tau}{H C} \\
& H=\frac{32 \pi^{3} n_{0}^{2}}{3 N \lambda^{4}}\left(\frac{n-n_{0}}{C}\right)^{2}
\end{aligned}
$$

$\tau$; excess turbidity, $M$; molecular weight, $C$; concentration in grams per $\mathrm{ml}, H$; a proportionality constant, $n$; refractive index of the solution, $n_{0}$; refractive index of the solvent, $N$; the Arogadto number, $\lambda$; wave length of the light used.

In the case of Amylose-I,

$$
\begin{array}{llllll}
C & 0.0333 & 0.0167 & 0.0083 & 0.0042 & 0.0021
\end{array}
$$

$\begin{array}{llllll}H C / \tau \times 10^{5} & 5.91 & 3.99 & 2.75 & 1.592 & 1.126\end{array}$

Therefore, $C \rightarrow 0 H C / \tau=0.67 \times 10^{-5} \mathrm{M} . \mathrm{W} .=140,000$

In the case of Retrograded Amylose-II,

$$
\begin{array}{llllll}
C & 0.0333 & 0.0167 & 0.0083 & 0.0042 & 0.0021
\end{array}
$$

$H C / \tau \times 10^{5} \quad 6.25 \quad 5.28 \quad 4.35 \quad(7.63)^{* * *}(29.3)^{* * *}$

Therefore, $C \rightarrow 0 H C / \tau=3.1 \times 10^{-5} \mathrm{M} . \mathrm{W} .=32,000$

7) P. Debye, J. Appl. Pbys., 17, 392 (1946).

* Phoenix's Differential Refractometer.

* * Brice Phoeaix's Instrument employed for measuring light scattering.

*** These values should be excluded from calculation because of the considerable infuence of the turbidity.

\section{DISCUSSION}

Heretofore, it has been shown by many workers that the average size of the amylose molecules plays an important role in the determination of the rate at which an amylose retrograded, but the actual molecular weight of amylose liable to retrograde has yet not been determined accurately.

From the experiments above mentioned, it became clear that retrograded amylose could be fractionated into two parts, one of which was extremely resistant to the amylase in the retrograded state, while the other was not.

The former fraction, resistant to the amylase, after being dissolved in alkaline solution and precipitated once more by neutralization, was found to be extremely resistant to the amylase as in the previous case. However, when it was crystallized as butanol complex, this amylose very liable to retrograde was found to be readily digested by the amylase. Molecular weight of this amylose $(32,000)$ was smaller than that of the original amylose $(140,000)$ when determined by using the light scattering method. This fact so far coincides well with suggestions of other workers.

Though many discussions $8,9,10,11$ appear about the glucosidic linkages of amylose molecules from the enzymatic degradation point of view, it should be considered that there is a certain molecular state of amylose, as described above, in which amylose retrogrades instantaneously and too rigidly to attack enzymatically.

As previously reported ${ }^{5 i}$, the authors observed the different digestibility of various native starch granules caused by black-koji amylase, which widely varied between two extremities, that is, potato starch which was most resistant to digestion and glutinous cereal starches which showed the lest resistance.

\footnotetext{
705 .

9) R. H. Hopkins and R. Bird, Nature, 172492 (1953).

10) A. Baba and H. Kojima, This Bulletin, 19, 167 (1955).

11) E. F. Neufeld and W.Z. Hassid, Arch. Biosbem. Biophys., 59,405 (1955).
} 
Considering these facts on the light of variability of amylose in the digestibility by the amylase as described above, it seems highly plausible to assume that the amount and, especially the nature of existanceof amylose would be one of the determining factors of resistance of raw starch to enzymic amylolysis.

\section{CONCLUSION}

(1) Amylose, in its retrograded form, was hydrolyzed by the black-koji amylase to glucose up to the extent of about 50 per cent with the exception of the indigestible part, the molecular weight of which was determined as 32,000 , while that of the original amylose as 140,000 .
(2) Notwithstanding the size of molecular weight, the amylose when crystallized as butanol complex was found to be hydrolyzed easily by black-koji amylase.

Acknowledgement The authors wish to thank Mr. K. Hayashi and Miss J. Oda for their invaluable advices through the determination of molecular weight of amylose by means of the light scattering method.

This work was aided in part by a grant from the Scientific Research Fund, Ministry of Education. (Composite research subject: Fundamental studies on the utilisation of starch. Representative: Zirô Nikuni) The authors' thanks are also due to Professor $\mathrm{Z}$. Nikuni.

[Bull. Agr. Chem. Soc. Japan, Vol. 22, No. 3, p. 162 168, 1958]

\title{
Geometrical Isomers of $\alpha$-Methylmuconic Acid
}

\author{
By Toshio Sugita, Yuzo Inouye and Minoru Ohno \\ Institute for Chemical Research, Kyoto University \\ Received January 6, 1958
}

\begin{abstract}
Peracetic acid oxidation of o-cresol gives $\alpha$-methyl-cis, cis-muconic acid (II), m.p. 189-90 This is lactonized with sulphuric acid to the lactonic acid (IV), which is converted to the lactonic methyl ester (V). The treatment of the lactonic ester with sodium methoxide affords (ס)-methyl ( $\alpha$ )-hydrogen $\alpha$-methyl-cis, trans-muconate (XI), which in turn is hydrolyzed to $\alpha$ methyl-cis, trans-muconic acid (XII), m.p. $172^{\circ}$ The cis, cis- and cis, trans-acids are isomerized to the trans, trans-isomer (XIV), m.p. $273^{\circ}$, by boiling with $20 \%$ aqueous sodium hydroxide. The geometrical configurations of these isomers are established by synthetic evidence and spectral data.
\end{abstract}

The authors have recently reported 1 ) on the geometrical configuration of $\alpha \delta$-dimethylsorbic acid. Now, the authors have investigated $\alpha$-methylmuconic acids as one of the polyene compounds of stereochemical interest. Kuhn and Michel2) prepared an isomer of

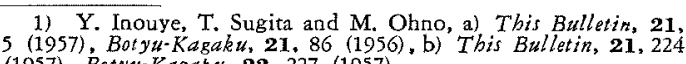
(1957), Botyu-Kagaku, 22, 227 (1957).

2) 'R. Kuhn and J. Michel, Ber., 71, 1119 (1938) $\alpha$-methylmuconic acid by the condensation of ethyl tiglate with ethyl oxalate, followed by acetylation and hydrolysis. However, the geometrical configuration of the acid has not yet been confirmed, though it is assumed tentatively to be trans-trans.

$\alpha$-Methylmuconic acid can exist theoretically in four geometrically isomeric forms cis-cis, 